\title{
Diabetic nephropathy is a risk factor for audio- vestibular system
}

\begin{abstract}
Objective: There is evidence that both inner ear and kidney tissue are immunological, biochemically and functionally related. Accordingly, this study was conducted to explore, if there is possible damage of the ear associated with diabetic nephropathy. This may help in early diagnosis and early management of hearing problem and the vestibular problem which is a leading cause of fall and death especially in the geriatric population.
\end{abstract}

Study design: Prospective study.

Methods: All participants subjected to the following, thorough history of audiovestibular symptoms, otoscopic examination, and basic audiological evaluation. Advanced audiological evaluation in the form of extended high frequency assessment. Distortion product otoacoustic emission (DPOAE) \& auditory brainstem response $(\mathrm{ABR})$. Vestibular assessment in the form of vestibular evoked myogenic potential (VEMP) and caloric test.

Results: The vestibular system more resistant to diabetes than the auditory system as the only affected part in this research is the inferior vestibular division while the superior division was not affected.

Conclusion: The audio-vestibular system was affected in patients with diabetic nephropathy more than the other diabetic patients and the vestibular system more resistant to diabetes than the auditory system. Screening of patients with diabetic nephropathy with a rapid test with minimal time consuming as OAE and cVEMP will help in early detection, early management of any affection of the audio-vestibular system and hence may aid in the prevention of imbalance, falls and death.

Keywords: diabetic nephropathy, vestibular system, hearing loss

\section{Introduction}

Diabetic nephropathy is damage to the kidneys caused by diabetes. In severe cases, it can lead to kidney failure. But not everyone with diabetes has kidney damage. ${ }^{1}$ There are no symptoms in the early stages. So it's important to have regular urine tests to find kidney damage early. Sometimes initial kidney damage can be reversed.

The problem is diagnosed by measuring albumin in the urine (micro or macroalbuminuria), this test called albumin/creatinine ratio (A/C ratio). Reijmer et al., ${ }^{2}$ reported that in the case of noncomplicated diabetics, the $\mathrm{A} / \mathrm{C}$ ratio was $0: 30 \mathrm{mg} / \mathrm{mg} / \mathrm{dl}$. However, in complicated diabetics it was $30: 300 \mathrm{mg} / \mathrm{mg} / \mathrm{dl}$ (microalbuminuria) or more than $300 \mathrm{mg} / \mathrm{mg} / \mathrm{dl}$ (macroalbuminuria).

There is evidence that both inner ear and kidney tissue are immunological, biochemically and functionally related. ${ }^{3}$ Accordingly, this study was conducted to explore, if there is possible damage of the ear associated with diabetic nephropathy, even there are no symptoms as regards hearing or balance disorder, and which part of the audiovestibular system affected more than the others and which part more resistant. This may help in early diagnosis and early management of hearing problem and a vestibular problem, which is a leading cause of falls and death especially in geriatric population. ${ }^{4}$

\section{Patients and methods}

Forty-two persons participated in this study, their age range from 20 to 52 years, 14 males and 28 females. They were divided into three groups, the control group consisted of 10 persons with no history of diabetes and the second group consisted of 17 diabetic patients with
Volume II Issue I - 2019

\author{
Rafeek Mohamed,' Hesham Mahmoud \\ Samy, ${ }^{2}$ Mohamed Ahmed Shaarawy, ${ }^{3}$ Kholoud \\ Nashaat Moustafa ${ }^{4}$ \\ 'Lecture of Audio,Vestibular Medicine, Minia University, Egypt \\ ${ }^{2}$ Assistant Professor of Audio Vestibular Medicine, Minia \\ University, Egypt \\ ${ }^{3}$ Professor of Internal Medicine, Minia University, Egypt \\ ${ }^{4}$ Resident Audio,Vestibular Medicine, Minia-University, Egypt
}

\begin{abstract}
Correspondence: Rafeek Mohamed MD, Lecture of AudioVestibular Medicine, Minia University, Borg-Elyasmen-Mogamaa elmahakem, Postal code 61 I I I, Egypt, Tel 01000432269, Email Rmalhfa2002@yahoo.com
\end{abstract}

Received: January 21, 2019 | Published: February 08, 2019 no diabetic nephropathy. The last group consisted of 15 diabetic patients complicated with diabetic nephropathy.

All participant had no complaint as regards hearing sensitivity or balance disorder and they were selected from patients referred to the Audio-Vestibular Unit at Minia University hospital, Egypt in the period from 6-2016 to 4-2017 while the control group were chosen from workers in the hospital. All participants in the study had been informed about the study aims and the detailed procedures to be used, before taking part. They gave written the ethical research committee approved consent for their participation in the study and all proceedings at Minia University.

All participants subjected to the following, thorough history of audio-vestibular symptoms, otoscopic examination, and basic audiological evaluation in the form of pure tone audiometry, speech audiometry, tympanometry, and acoustic reflex recording. Advanced audiological evaluation in the form of extended high frequency assessment in the frequency range $(9 \& 10 \& 11,200 \& 12,500 \& 14$ $\& 18 \& 20 \mathrm{KHz}$ ). Distortion product otoacoustic emission (DPOAE) \& auditory brainstem response (ABR). Vestibular assessment in the form of vestibular evoked myogenic potential (VEMP) and caloric test.

The basic audiological evaluation was done in the form of pure tone audiometry (PTA) and speech audiometry, using Amplaid 309 audiometer (Amplus Corp. Boulder, CO, U.S.A.) and sound treated room (amplisilence) to assess hearing sensitivity. Air conduction threshold was obtained for the frequency range $250-8000 \mathrm{~Hz}$ at single octave intervals using a TDH 49 earphone (Telephonics Corporation, Farm ingdale, NY, U.S.A.). Bone conduction threshold was obtained 
for the frequency range $500-4000 \mathrm{~Hz}$ at single octave intervals using a B71 bone vibrator (Radioear, New Eagle, PA, and the U.S.A.). Speech reception threshold (SRT) and speech discrimination score (SDS) using bisyllabic and monosyllabic phonetically balanced word respectively were determined.

Advanced audiological evaluation in the form of HFA (extended high-frequency audiometry). It can assess hearing in the frequency range $(9 \& 10 \& 11,200 \& 12,500 \& 14 \& 18 \& 20 \mathrm{KHz})$. DPOAE using the Intelligent Hearing system (IHS) with Smart OAE 4.5 software. Two tones were used: $\mathrm{L} 1=65 \mathrm{~dB}$ SPL and $\mathrm{L} 2=55 \mathrm{~dB}$ SPL, while $\mathrm{f} 2$ f1 was 1.22. Both the amplitude of the response of the distortion product (DP) at 2f1-f2 and background noise (Ns) were obtained at nine points corresponding to $\mathrm{f} 2$ frequencies of $553,783,1105,1560$, 2211, 3125, 4416, 6250 and $8837 \mathrm{~Hz}$. These measurements were used to build a DP-gram by displaying the DP against the $\mathrm{f} 2$ frequency. The signal to noise ratio (SNR) was measured (SNR=DP-Ns) at each of these nine points. DPOAE was considered pass, thereby reflecting normal cochlear function, if the SNR was $>3 \mathrm{~dB}$ SPL on at least $70 \%$ of the tested frequencies. If SNR was > 3dB SPL in less than $70 \%$ of the tested frequencies but at least at one tested frequency, it was considered a partial pass. If SNR $<3 \mathrm{~dB}$ SPL in all tested frequency, the result was considered failed.

Auditory Brain Stem Response (ABR); ABR was done using intelligent hearing system (IHS) with smart evoked potentials software version 4.5 . The stimuli were $100 \mu$ s alternating click delivered through insert earphone at $80 \mathrm{~dB} \mathrm{nHL}$. Repetition rate of the stimuli was $21.1 \mathrm{p} / \mathrm{s}$ and $71.1 \mathrm{p} / \mathrm{s}$. Electrode montage was high forehead to ipsilateral mastoid. The common electrode was placed on the contralateral mastoid. The response was filtered between 100 and $3000 \mathrm{~Hz}$, amplified 100,000 times, recorded over $10.24 \mathrm{~ms}$ time window, and 2000 sweeps were averaged for each run. Two runs were recorded at a high repetition rate and low repetition rate in each ear to assure repeatability. Parameters analyzed were; Absolute peak latency\& interpeak latency of wave (I\&III and V) and interaural peak latency difference for waves III and V. Latency shift for wave V at high repetition rate versus low repetition rate was also assessed.

Vestibular assessment in the form of vestibular evoked myogenic potential (VEMP) was performed using IHS with Smart EP software, version 4.5. The patients were tested either in the sitting position or in the supine position, and head rotated away from the stimulated side. The patients were instructed to tense the sternocleidomastoid muscle during acoustic stimulation and relax it between recording sessions. The electrode montage to record the electromyographic activity was as following an ipsilateral surface electrode (active) on the middle of the sternocleidomastoid muscle, a reference electrode on the ipsilateral sternoclavicular joint and a ground electrode on the forehead. The stimulus was $500 \mathrm{~Hz}$ tone burst $(1 \mathrm{~ms}$ rise and fall time and $5 \mathrm{msec}$ plateau time). The stimulus was presented ipsilaterally through Telephonics TDH-49 headphone. The intensity of each stimulus was $95 \mathrm{~dB}$ nHL. The electromyographic signal was amplified (5000 times) and bandpass filtered $(10-1500 \mathrm{~Hz})$. The repetition rate of the stimuli was 5.1 stimulus/second, and an average of 200 sweeps was taken, and two waveforms were recorded to test wave repeatability. The analysis window started $30 \mathrm{~ms}$ before stimulus onset and ended $70 \mathrm{~ms}$ after stimulus onset (i.e., from-30 msec to $70 \mathrm{msec}$ ). Upon recording the waveforms, the first positive deflection was marked as P1, and the first negative deflection was marked as N1. Such wave (P1-N1) was the wave to be analyzed. Peak to peak P1-N1 corrected amplitude (CA), P1 latency and N1 latency were calculated for both ears. CA was calculated according to such formula:

$$
\frac{P 1-N 1 \text { amplitude }}{\text { Root means square of } 30 \mathrm{~ms} \text { before stimulus onset }}
$$

Asymmetric ratio (AR) was calculated according to such method:

(Larger corrected amplitude - smaller corrected amplitude)/ (larger corrected amplitude + smaller corrected amplitude) $\times 100 .^{5}$

Reduced amplitude was diagnosed when AR was $>37 \%$. Abnormal P1 latency and N1 latency were diagnosed when each was larger than mean $+2.5 \mathrm{SD}$ of the clinic normative values.

\section{Results}

All participants in this research had no complaint as regards hearing sensitivity so in basic audiological evaluation (PTA and speech discrimination) reveal that there is no statistically significant difference between all groups as shown in Table 1 and Table 2 .

However, in advanced audiological evaluation as in HFA\& $\mathrm{OAE}$ and $\mathrm{ABR}$, there is a statistically significant difference in the complicated group more than the others as shown in Table 3-6.

Although nearly all the auditory system either the peripheral part of the central auditory brainstem pathway was affected in the complicated diabetic patients, the vestibular system more resistant to diabetes than the auditory system as the only affected part in this research is the inferior vestibular division, affected only in amplitude while latency not changed and the superior division also not affected as reflected from caloric test, Table $7 \&$ Table 8.

Table I Comparison between the studied groups as regarding as regarding PTA

\begin{tabular}{lllll}
\hline PTA & Controls & Non-complicated DM & Complicated DM & P \\
\hline 250 & $19.2 \pm 0.7$ & $18.5 \pm 1.6$ & $18.1 \pm 1.8$ & 0.07 \\
500 & $18.5 \pm 1.5$ & $18.4 \pm 0.9$ & $17.3 \pm 0.8$ & 0.09 \\
1000 & $17.5 \pm 2.1$ & $18.8 \pm 1.3$ & $18.5 \pm 1.4$ & 0.07 \\
2000 & $17.5 \pm 2.6$ & $18.8 \pm 1.3$ & $17.1 \pm 1.5$ & 0.08 \\
4000 & $18 \pm 1.1$ & $18.1 \pm 1.8$ & $18.6 \pm 1.2$ & 0.09 \\
8000 & $17 \pm 1.1$ & $18.2 \pm 6.4$ & $17.8 \pm 1.1$ & 0.07 \\
\hline
\end{tabular}


Table 2 Comparison between the studied groups as regarding speech discrimination

\begin{tabular}{lllll}
\hline Speech discrimination & Controls & Non-complicated DM & Complicated DM & P \\
\hline Range & 100 & $96-100$ & $96-100$ & 0.07 \\
Mean \pm SD & 100 & $99.2 \pm 1.5$ & $98.5 \pm 1.9$ & \\
\hline
\end{tabular}

Table 3 Comparison between the studied groups as regarding extended high frequency

\begin{tabular}{|c|c|c|c|c|}
\hline $\begin{array}{l}\text { Extended high } \\
\text { frequency }\end{array}$ & Controls & Non-complicated DM & Complicated DM & $\mathbf{P}$ \\
\hline 9000 & $31.7 \pm 7.1$ & $41.6 \pm 9.1$ & $41.5 \pm 7.3$ & $0.004 *$ \\
\hline 10000 & $33 \pm 7.8$ & $44.7 \pm 12.1$ & $48.8 \pm 8.2$ & $0.001 *$ \\
\hline 11200 & $40 \pm 8.5$ & $57.6 \pm 17.5$ & $71.3 \pm 9.8$ & $0.001^{*}$ \\
\hline 12500 & $50.5 \pm 8.6$ & $73.5 \pm 21.3$ & $93.1 \pm 10.2$ & $0.001 *$ \\
\hline 14000 & $62.1 \pm 9.1$ & $95.8 \pm 18.7$ & $107.8 \pm 25.6 .1$ & $0.001 *$ \\
\hline 16000 & $86.7 \pm 11.6$ & $105.7 \pm 9.9$ & $108 \pm 2.3$ & $0.001 *$ \\
\hline 18000 & $106.7 \pm 3.7$ & $109.5 \pm 1.3$ & $110 \pm 1.3$ & $0.001 *$ \\
\hline
\end{tabular}

Table 4 Post Hoc test for comparison between each two groups as regarding extended high frequency

\begin{tabular}{llll}
\hline $\begin{array}{l}\text { Extended high } \\
\text { frequency }\end{array}$ & $\begin{array}{l}\text { Controls vs. Non- } \\
\text { complicated }\end{array}$ & $\begin{array}{l}\text { Controls vs. } \\
\text { complicated DM }\end{array}$ & $\begin{array}{l}\text { Non complicated vs. } \\
\text { complicated DM }\end{array}$ \\
\hline 9000 & $0.002^{*}$ & $0.002^{*}$ & $0.04^{*}$ \\
10000 & $0.005^{*}$ & $0.001^{*}$ & $0.02^{*}$ \\
11200 & $0.002^{*}$ & $0.002^{*}$ & $0.006^{*}$ \\
12500 & $0.001^{*}$ & $0.001^{*}$ & $0.001^{*}$ \\
14000 & $0.001^{*}$ & $0.001^{*}$ & $0.01^{*}$ \\
16000 & $0.001^{*}$ & $0.001^{*}$ & $0.03^{*}$ \\
18000 & $0.001^{*}$ & $0.001^{*}$ & $0.04^{*}$ \\
\hline
\end{tabular}

Table 5 Comparison between the studied groups as regarding OAE

\begin{tabular}{|c|c|c|c|c|}
\hline OAE & Controls & Non complicated DM & Complicated DM & $\mathbf{P}$ \\
\hline \multirow[t]{4}{*}{1000} & -13.25 & -19 & -55.5 & $\mathrm{P}=0.03 *$ \\
\hline & $8.5 \pm 4.1$ & $8.5 \pm 5.1$ & $-0.45 \pm 13.5$ & $\mathrm{P} 1=0.9$ \\
\hline & & & & $\mathrm{P} 2=0.03$ \\
\hline & & & & $\mathrm{P} 3=0.01 *$ \\
\hline \multirow[t]{4}{*}{2000} & -33 & $9.3-23.1$ & -21.5 & $\mathrm{P}=0.03 *$ \\
\hline & $11.1 \pm 8.8$ & $15.1 \pm 4.7$ & $9.3 \pm 5.3$ & $\mathrm{P} 1=0.1$ \\
\hline & & & & $\mathrm{P} 2=0.4$ \\
\hline & & & & $\mathrm{P} 3=0.01 *$ \\
\hline \multirow[t]{4}{*}{4000} & $3.7-19.4$ & -24.2 & -26.2 & $\mathrm{P}=0.001 *$ \\
\hline & $15.4 \pm 4.7$ & $11.01 \pm 6.1$ & $5.3 \pm 6.4$ & $\mathrm{P} 1=0.07$ \\
\hline & & & & $\mathrm{P} 2=0.001 *$ \\
\hline & & & & $\mathrm{P} 3=0.01 *$ \\
\hline \multirow[t]{4}{*}{6000} & -18.8 & -14.15 & -12.75 & $\mathrm{P}=0.003^{*}$ \\
\hline & $9.5 \pm 5.9$ & $4.2 \pm 4.5$ & $2.8 \pm 3.7$ & $\mathrm{P} 1=0.006^{*}$ \\
\hline & & & & $\mathrm{P} 2=0.001 *$ \\
\hline & & & & $\mathrm{P} 3=0.04 *$ \\
\hline
\end{tabular}

$\mathrm{P}$, comparison between all three groups; $\mathrm{pl}$, comparison of controls versus none complicated; $\mathrm{P} 2$, controls versus complicated ; 3 , none complicated versus complicated. 
Table 6 Post Hoc test for mean and SD comparison between each two groups as regarding ABR

\begin{tabular}{lllll}
\hline ABR(latencies in ms) & $\begin{array}{c}\text { Mean } \pm \text { SD } \\
\text { Controls }\end{array}$ & Non-complicated DM & Complicated DM & P value \\
\hline Wave I latency & $1.4 \pm 0.1$ & $1.5 \pm 0.09$ & $1.5 \pm 0.1$ & $\mathrm{P} 1=0.06$ \\
& & & & $\mathrm{P} 2=0.07$ \\
Wave III latency & $3.5 \pm 0.2$ & $3.6 \pm 0.1$ & $3.7 \pm 0.1$ & $\mathrm{P} 1=0.3$ \\
Wave V latency & $5.4 \pm 0.2$ & $5.5 \pm 0.1$ & & $\mathrm{P} 2=0.01^{*}$ \\
& & & $5.8 \pm 0.2$ & $\mathrm{P} 1=0.2$ \\
\end{tabular}

PI, comparison of controls versus none complicated; P2, controls versus complicated.

Table $7 \mathrm{PI}-\mathrm{NI}$ latencies and amplitude in the studied groups.

\begin{tabular}{lllll}
\hline CVMP & Controls & Non-complicated DM & Complicated DM & P \\
\hline P1 & $15.3-20.8$ & $15.3-24.3$ & $14.3-24.8$ & $\mathrm{P}=0.2$ \\
& $17.5 \pm 1.8$ & $19.2 \pm 2.3$ & $19.4 \pm 3.4$ & $\mathrm{P} 2=0.1$ \\
& & & $\mathrm{P} 3=0.8$ \\
N1 & & & $\mathrm{P}=0.3$ \\
& & & $23-35.8$ & $\mathrm{P} 1=0.1$ \\
& $20-30$ & $22.5-34.5$ & $28.2 \pm 3.8$ & $\mathrm{P} 2=0.1$ \\
& & & $\mathrm{P} 3=0.9$ \\
N1P1Lat & $4.7-10.3$ & $4-14.5$ & & $\mathrm{P}=0.9$ \\
& $8.6 \pm 1.6$ & $8.8 \pm 2.4$ & $4.1-12.3$ & $\mathrm{P} 1=0.8$ \\
& & & $8.5 \pm 2.3$ & $\mathrm{P} 2=0.8$ \\
& & & & $\mathrm{P} 3=0.7$
\end{tabular}

$\mathrm{P}=$ comparison between all three groups, $\mathrm{pl}=$ comparison of controls versus none complicated. $\mathrm{P} 2=$ controls versus complicated and $\mathrm{p} 3=$ none complicated versus complicated.

Table 8 Comparison between the studied groups as regarding caloric test (Slow Phase Velocity-SPV)

\begin{tabular}{lll}
\hline Non complicated DM & complicated DM & P \\
\hline $\mathrm{N}=17$ & $\mathrm{~N}=15$ & \\
$18.6-44.6$ & $12-57.5$ & 0.5 \\
$28.7 \pm 8.3$ & $31.03 \pm 12.4$ & \\
\hline
\end{tabular}

\section{Discussion}

There is evidence that both inner ear and kidney tissue are immunological, biochemically and functionally related Egberts AC et al., ${ }^{3}$ what about the ear in complicated diabetic cases with diabetic nephropathy. Accordingly, this study was conducted to explore, if there is possible damage of the ear associated with diabetic nephropathy, even there are no symptoms as regards hearing or balance disorder, and which part of the audio-vestibular system affected more than the others and which part more resistant. This may help in early diagnosis and early management of hearing problem and a vestibular problem which is a leading cause of death especially in the geriatric population. ${ }^{4}$

All participants in this research had no complaint as regards hearing sensitivity or balance problem. All of them had standard basic audiological evaluation either the pure tone audiometry or speech discrimination. However, in advanced audiological evaluation which assess the peripheral auditory system as OAE and HFA or more proximally the auditory brainstem pathway which is examined by 
ABR showing affected results in group with diabetic nephropathy, either in the form of absent response in OAE, impaired hearing threshold in HFA and prolonged absolute latencies in ABR waves in patients with diabetic nephropathy. These results were matched was Dennis et al., ${ }^{6}$ reported that high frequency $(9000-20000 \mathrm{HZ})$ threshold values from the diabetic group with standard conventional audiometry results were higher than the high-frequency thresholds from the control group.

Hearing impairment has been reported to be one of the late complications of diabetes mellitus (DM), and the frequency varies. Many authors have tried to identify the underlying cause of hearing loss in diabetic patients. In conclusion, the effective mechanisms are microangiopathy of the inner ear, neuropathy of the auditory nerve, or combination of both, outer hair cell dysfunction and disruption of endolymphatic potential. ${ }^{7}$

All diabetic patients with diabetic nephropathy had absent response in $\mathrm{OAE}$ and prolonged absolute latencies in ABR which is matched with The data collected from Ferenc, et al. ${ }^{8}$ Study support the hypothesis that long-standing diabetes mellitus and diabetic complications might be revealed as a cause of specific dysfunctions of the central auditory pathways and prolongation of latency of ABR in patients with DM should alert us to possible damage to the auditory nerve, and close follow-up is needed in those patients.

A study by Francesco Ottaviani, et al., ${ }^{9}$ analyses otoacoustic emission (OAEs) on normal hearing subjects with diabetes and on controls. Patients who some of them had signs of complications of diabetes, were studied. Their findings were failed otoacoustic emissions in diabetic patients with complications than diabetic patients without complications in spite of a normal audiometric hearing threshold. Francesco Ottaviani et al., ${ }^{9}$ in their study suggests that cochleopathy can be detected in a relatively high proportion of subjects with diabetes.

Although, nearly all parts of the auditory system are affected in patients with diabetic nephropathy, either the peripheral auditory system (cochlea) or more proximal pathway as auditory brainstem pathway, not all parts of the vestibular system is affected. The superior vestibular division not affected even with diabetic nephropathy as reflected from the caloric test. However, the inferior vestibular division is affected as reflected from altered amplitude in cVEMP.

Behnoush Kamali et al., ${ }^{10}$ reported in a comparative crosssectional study. Including diabetic patients without complications and other diabetic patients with complications. There was a significant difference between the groups in $\mathrm{P} 1, \mathrm{~N} 1$ latency $(\mathrm{P}<0.05)$ and no significant difference between the groups in absolute or relative amplitudes. Indicating vestibular dysfunction and suggest a pathology in the saccule or its innervating neurons. This study is controversial with our research as we found no significant difference as regarding latency of cVEMP (P1, N1, and P1-N1 latency) in complicated and non-complicated diabetics. On the other hand, when looking at p1N1amplitude of cVEMP, we found a significant reduction of $\mathrm{p} 1-$ $\mathrm{N} 1$ amplitude in diabetic patients with complications ( $\mathrm{p}$ value $=0.01$ ) than in people with diabetes without complications.

It was hypothesized that innervating neurons of vestibular nerve remains non-pathological in individuals with diabetes mellitus as the auditory brainstem responses were present, usually implying normal functioning of the cochlear nerve fibers. And since cochlear and vestibular nerve, are part of the same 8th cranial nerve, hence chances of presence of a lesion in vestibular nerve reduces.And this like what happened in our current study in non-complicated group as ABR and cVEMP were not affected but after occurrence of complication of diabetes and prolonged duration of disease, we found abnormal ABR and reduced cVEMP amplitude, suggesting Subclinical saccular affection can be a newly defined diabetes-related complication Wang, et al. ${ }^{11}$

Karlin Fabianne et al., ${ }^{12}$ have suggested that recently diagnosed DM patients show no vestibular test abnormalities. They raised the hypothesis that the effect of DM on vestibular function might be caused by complications such as diabetic neuropathy and microangiopathy, which are absent in initial phases of the disease.

In a study group included 30 patients, with diabetes mellitus, it was reported that no subjective dizziness and no abnormalities regarding video-nystagmography were noted in all tested patient Lasisi et al. ${ }^{13}$ In our current study show the same results as Lasisi study. We found in all diabetic patients ears; There was no significant difference between both ears and no significant finding in effects as regarding slow phase caloric test $(\mathrm{p}=0.5)$ and no correlation between slow phase caloric test and diabetic complications.

The overall results were reflecting that the auditory system affected more than the vestibular system. The superior vestibular nerve division was more resistant to diabetes complication than the inferior division. Testing of patients with diabetic nephropathy even with a rapid test with minimal time consuming as OAE and cVEMP will help in early detection of audio-vestibular system affection and hence for more investigation of the audio-vestibular system or follow up if it was free.

\section{Summary}

The audio-vestibular system was affected in patients with diabetic nephropathy more than the other diabetic patients.

The vestibular system more resistant to diabetes than the auditory system. Screening of patients with diabetic nephropathy with a rapid test with minimal time consuming as OAE and cVEMP will help in early detection, early management of any affection of the audiovestibular system which may help in prevention of balance problem, falling and death specially in geriatric population.

\section{Acknowledgments}

None.

\section{Conflicts of interest}

The author declares there is no conflict of interest.

\section{References}

1. American Diabetes Association. Standards of medical care in diabetes-2015. Diabetes Care. 38(Suppl 1):S1-S93.

2. Reijmer YD, Van den Berg E, Breedijk MA, et al. On behalf of the Utrecht Diabetic Study Group Department of Neurology. 2010.

3. Verdel BM, van Puijenbroek EP, Souverein PC, et al. Drug-related nephrotoxic and ototoxic reactions: a link through a predictive mechanistic commonality. Drug Saf. 2008;31(10):877-884.

4. Crawford. Balance Problems: A Leading Cause of Death. Health Capsule Articles. 2016. 
5. Gary P, Jacobson, Neil TS. Balance Function Assessment and Management. Second Edition. 2008.

6. Ahmed HO, Dennis JH, Badran O, et al. High-frequency $(10-18 \mathrm{kHz})$ hearing thresholds: reliability, and effects of age and occupational noise exposure. Occup Med. 2001;51(4):245-258.

7. Venkata K, Robert S, Hinrich S. The effect of diabetes on sensorineural Hearing Loss. Otology\& Neurotology. 20003;24(3):382-286.

8. Ferenc T, Tamas TV, Laszl R, et al. Investigation of Auditory Brainstem Function in Diabetic Patients. International Tinnitus Journal. 2003;9(2):84-86.

9. Ottaviani F, Dozio N, Neglia CB, et al. Absence of otoacoustic emissions in insulin-dependent diabetic patients: is there evidence for diabetic cochleopathy? J Diabetes Complications. 2002;16(5):338-343.
10. Kamali B, Hajiabolhassan F, Fatahi J, et al. Effects of Diabetes Mellitus with or without Neuropathy on Vestibular Evoked Myogenic Potentials. Act Medica. 2013;51(2):107-112.

11. Wang SJ, Yeh $\mathrm{TH}$, Chang $\mathrm{CH}$, et al. Consistent latencies of vestibular evoked myogenic potentials. Ear Hear. 2008;29(6):923-929.

12. Klagenberg KF, Zeigelboim BS, Jurkiewicz AL, et al. Vestibulocochlear manifestations in patients with types I diabetes mellitus. Braz $J$ Otorhinolaryngol. 2007;73(3):353-358.

13. Lasisi OA, Nwaorgu OG, Bella A, et al. Cochleovestibular complications of diabetes mellitus in Ibadan, Nigeria. International Congress Series. 2005; 1240:1325-1328. 